\title{
The Presence of Higher-Order Laue Zone Intensities and the Relrod Effect in Cubic Metals in the Transmission Electron Microscope
}

\author{
Cody Miller, Robert Field and Michael Kaufman \\ Colorado School of Mines, Golden, CO, USA
}

The presence of $1 / 3\{422\}$ reflections in $<111>$ selected area diffraction patterns (SADP) has been attributed to short-range order (SRO) leading to diminished stress corrosion cracking resistance in IN690 after extended aging ${ }^{1}$. In this study, it is shown that these diffuse intensities in IN690 are likely the result of scattering from higher-order Laue zone (HOLZ) reflections into the positions observed in the zero order Laue zone (ZOLZ). To support this hypothesis, it is shown that several fcc structures, such as IN600, IN945, commercially pure (CP) nickel, Co-28.5Cr-6Mo and Ni-33at\%Cr as well as diamond cubic structures such as $\mathrm{Si}$ and zinc-blende structures (CdTe) also give rise to intensities in these positions. This hypothesis explains the presence of diffuse scattering down the $<111>$, in addition to several other higher index zone axes.

The diffuse intensities originally investigated in IN690 (Figure 1) have been confirmed in the above mentioned systems. Centered dark-field imaging shows no indication of ordered domains. A focused ion beam (FIB) liftout of CP nickel was examined to ensure this diffuse scattering was not an artifact of sample preparation or oxidation. Cryo-stage TEM was performed on IN690 to rule out thermal diffuse scattering (TDS) as a possible cause. Additionally, similar results have been observed in silicon nanowires, as shown by Bell and coworkers, which the authors attribute to HOLZ diffraction via the relrod effect. ${ }^{1}$

To further explain this phenomenon, an experiment was conducted on a Co-28.5Cr-6Mo alloy, shown in Figure 2. Tilting from an adjacent [112] zone axis, intensity from the (111) reflections is seen to be sustained into the $1 / 3\{422\}$ position within the [111] zone. Further examining the remaining two [112] zone axes near the [111], we can explain all diffuse intensities present in the [111] zone axis via HOLZ diffraction. Furthermore, simulations via JEMS crystallography software demonstrate that HOLZ effects can account for all anomalous intensities in the SADP, as shown in Figure $3 .^{2}$

The concept of HOLZ diffraction in this context requires that this effect occur from layers both above below the zero-order Laue zone (ZOLZ). Furthermore, a full understanding of the Weiss zone law is required. For zone axes where $\mathrm{U}+\mathrm{V}+\mathrm{W}$ sums to an odd value, first-order Laue zone (FOLZ) diffraction occurs. Conversely, for the case when $\mathrm{U}+\mathrm{V}+\mathrm{W}$ sums to an even value, second-order Laue zone (SOLZ) diffraction occurs. Geometrical considerations of both the Ewald's sphere and HOLZ reflections within the reciprocal lattice show that these events are likely to result in diffuse intensity projected into the ZOLZ layer, with modeling of this diffraction event currently in progress.

[1] Y. S. Kim, "Effect of short-range ordering on stress corrosion cracking susceptibility of Alloy 600 studied by electron and neutron diffraction,” Acta Mater., vol. 83, pp. 507-515, 2015.

[2] D. C. Bell, Y. Wu, C. J. Barrelet, S. Gradecak, J. Xiang, B. P. Timko, and C. M. Lieber, "Imaging and analysis of nanowires.," Microsc. Res. Tech., vol. 64, no. 5-6, pp. 373-89, Aug. 2004.

[3] P. Stadelmann, “JEMS EMS Java Version V4,” 2014. [Online]. Available:

http://cimewww.epfl.ch/people/stadelmann/jemsWebSite/jems.html. 


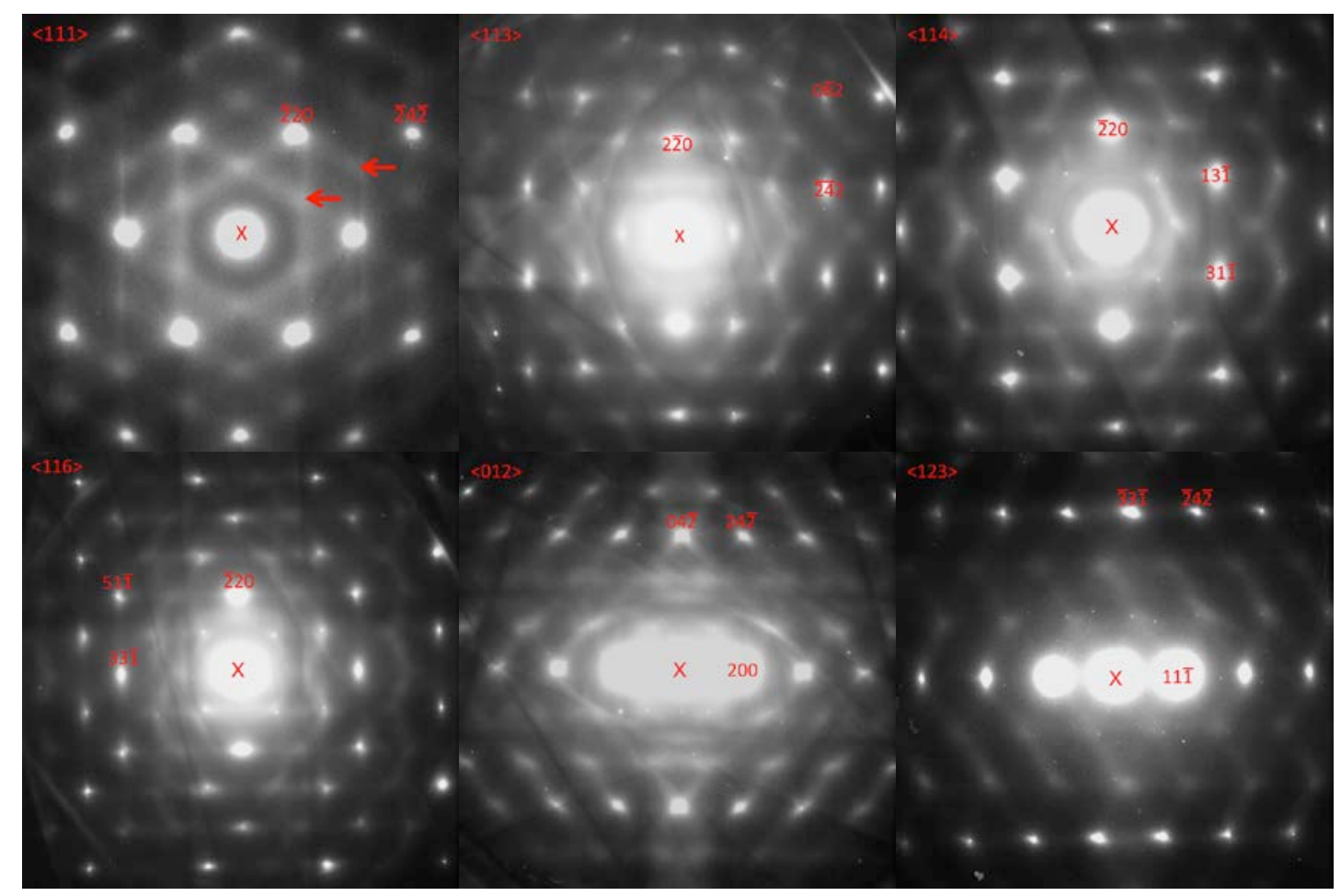

Figure 1. SADP's of IN690, showing additional diffuse intensities down multiple zone axes, all of which are attributable to first- and second-order Laue zone diffraction.
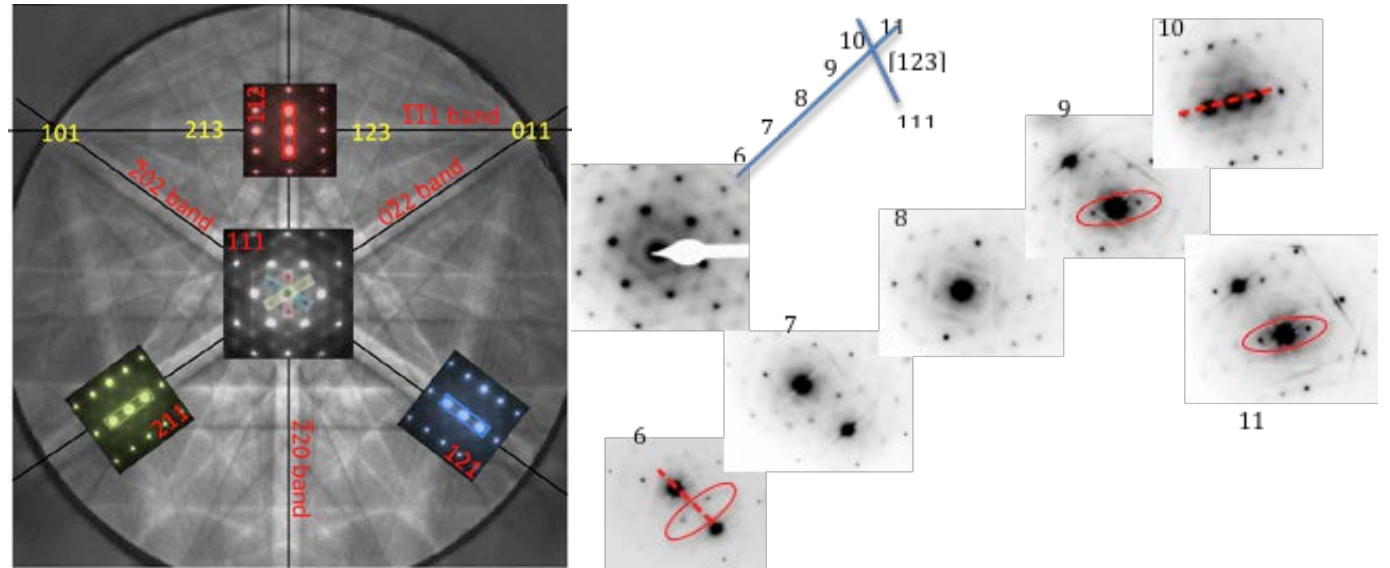

Figure 2. SADP's of Co-28.5Cr-6Mo tilting experiment, showing incomplete cancellation of $\{111\}$ reflections within the $<111>$ zone axis.

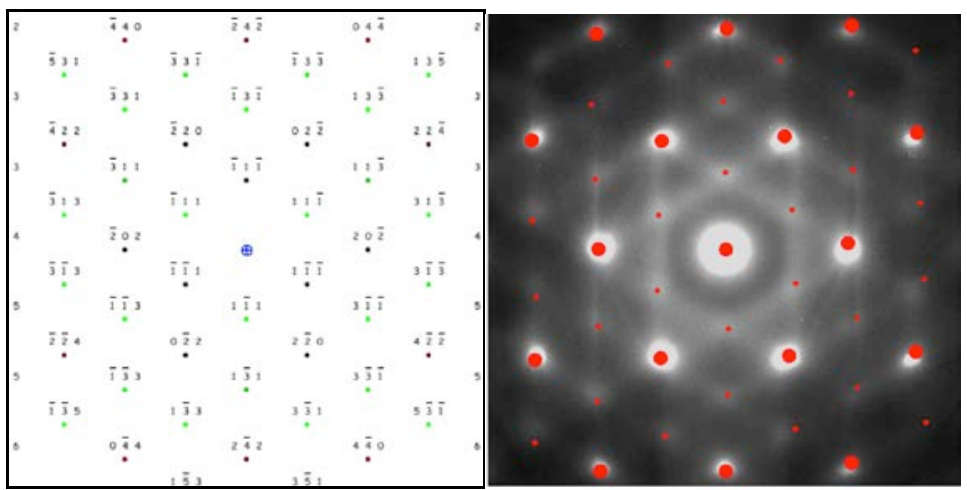

Figure 3. JEMS simulation of HOLZ reflections in the 111 ZA (left), overlaid on a [111] SADP (right). 